\title{
Teaching Electromagnetics via Virtual Experiments
}

\author{
Poman So \\ Department of Electrical Engineering \\ University of Victoria, Victoria, BC, Canada \\ Poman.So@ECE.UVic.CA
}

\begin{abstract}
Learning electromagnetic field theory through abstract mathematical equations alone is a challenging task to most students. They look upon the subject as a difficult, abstract, and highly mathematical matter. The author has thus developed a modeling and simulation tool with special features designed for teaching electromagnetic field theory. The software tool has advanced features and field animation capabilities to transform abstract electromagnetic field theory concept into realistic images on the computer screen.
\end{abstract}

\section{Introduction}

Electromagnetic modeling software packages are good tools for demonstrating electrodynamic phenomena such as wave propagation, reflection, refraction, scattering and radiation. Due to the high cost of software development, virtually all good commercially available packages are developed for the engineering industries. Most students would find these software packages difficult to use. The author has thus developed a software tool for teaching engineering electromagnetics. The tool is based on a field modeling package that has more than twenty years of development history [ $1-4]$. The author has been using the tool in teaching for years; experience has shown that and most students can master the graphical user interface of the software in just one laboratory session. The tool is called $\mathcal{M E F}$ iSTo-3D $\mathcal{N}$ ova; it is a light-weighted version of $\mathcal{M E F}$ FiSTo-3D Pro. In terms of features, these two software packages are virtually identical. Henceforth, to avoid unnecessary distinction between the two slightly different products, the author would just use the name $\mathcal{M E F}$ F $S \mathcal{T}$ o when referring to the software. $\mathcal{M E F}$ S $S \mathcal{T}$ o-3 $\mathcal{D} \mathcal{N}$ ova is free, therefore it a good software tool for students to investigate dynamic electromagnetic phenomena on their home computers.

In the following sections a sequence of numerical experiments that demonstrates some fundamental behaviour of electromagnetic fields and waves will be presented. The first set of experiments illustrates the time varying circulating behaviour of electromagnetic fields as described by Maxwell's equations; the integral form of Faraday's and Ampere's laws can be validated using the software's integrator. The second set of experiments deal with wave propagation, reflection and transmission. Finally, advanced simulation examples that illustrate the negative phase velocity and focusing capability of metamaterial are presented before the conclusion.

\section{Using $\mathcal{M E F}$ FiSTo}

Before jumping into the details of the numerical experiments, it is necessary to give a short description on using the software. $\mathcal{M E F}$ F $\mathcal{S}$ To is a general purpose field simulation package. The software has no application wizard to create "canned" structures; all structures to be simulated must be created with the built-in geometry primitives - namely the line, arc, rectangle, plane, box and cylinder. Material properties are associated with the elements at the object creation time. Figure 1 depicts the main window of $\mathcal{M E F i S \mathcal { T } o}$ together with an editor child window. The toolbar at the top contains geometry primitives. The Drawing Object selection list at the left panel displays various primitive attributes while the Computation Object list shows the elements comprising the structure to be modeled. To create the hollow three-dimensional metallic object as shown in figure 1 , one needs to select the perfect electric attribute on the left panel and select the rectangle primitive on the horizontal toolbar; these selections are highlighted in the figure. The next step is to specific the coordinates of two vertices that define the corners of the object. This can be done by clicking at two different $y$-locations at the $x y$-pane, i.e. specify $y 1$ and $y 2$, and then draw a rectangle at the $x z$-pane. This sequence of mouse operations specify two corners, namely $(x 1, y 1, z 1)$ and $(x 2, y 2, z 2)$, and an up-vector, $(0,1,0)$ for the rectangular object. Complex objects are built by assembling a collection of simple primitives. It takes a few trail and error manipulations to get acquainted with this object creation procedure. 


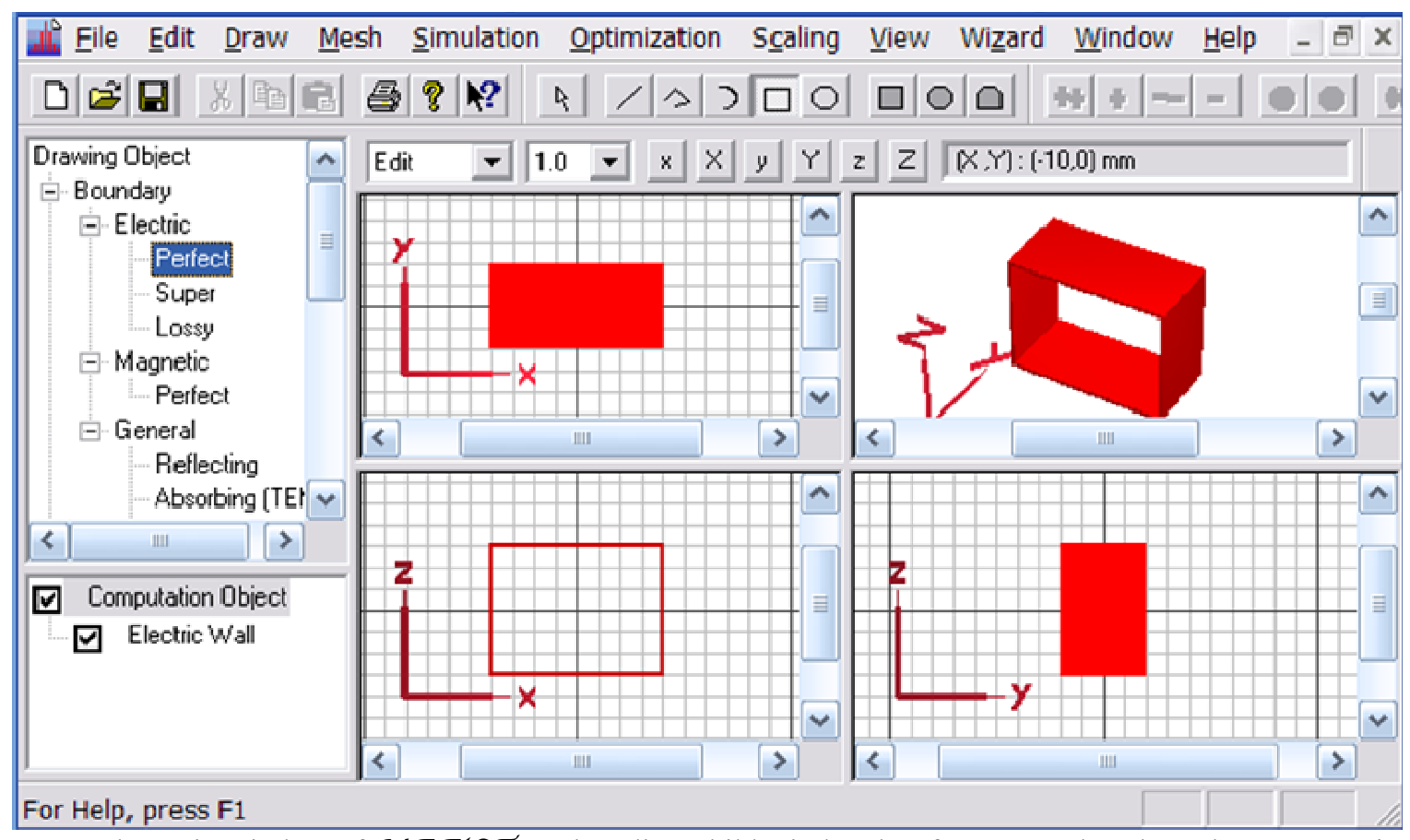

Figure 1: The main window of $\mathcal{M E F} i \mathcal{S} \mathcal{T}$. The editor child window has four panes that show the cross-sectional and three-dimensional views of a hollow rectangular object.

\section{The Experiments}

\subsection{Faraday's and Ampere's Laws}

The first experiment illustrates the Faraday's Law. The simplest way to simulate this field behaviour is to excite a magnetic field polarized in the $z$-direction in a magnetic cavity, figure 2 . The cavity can be excited either with the fundamental field distribution (half-sin in the $x$ and $y$ directions) or with a transient signal. The latter one is more general because it can be used to excite cavities of arbitrary shapes. Also shown in figure 2 is a transient signal that contains the resonant frequency of the cavity $\left(f_{\mathrm{o}}=10.59 \mathrm{GHz}\right)$. As the field resonating inside the structure, the circulating and time-varying behaviour of $\mathrm{E}$ and $\mathrm{H}$ can be easily seen. A snapshot of the $\mathcal{M E F}$ FiSTo field animation is shown in figure 3. The dynamic field images can only show $\mathrm{E}$ and $\mathrm{H}$ are varying in time with $\mathrm{E}$ circulating on the $x y$-plane, it is impossible to conclude $\nabla \times E=-\frac{\partial B}{\partial t}$ However, the integral form of Faraday's Law, $\oint E \cdot d l=-\frac{d}{d t} \int B \cdot d s$, can be validated easily by $\mathcal{M E F} \dot{S} \mathcal{T}_{0}$ 's integrator windows. Ampere's Law can be simulated in a similar manner using an electric resonator.

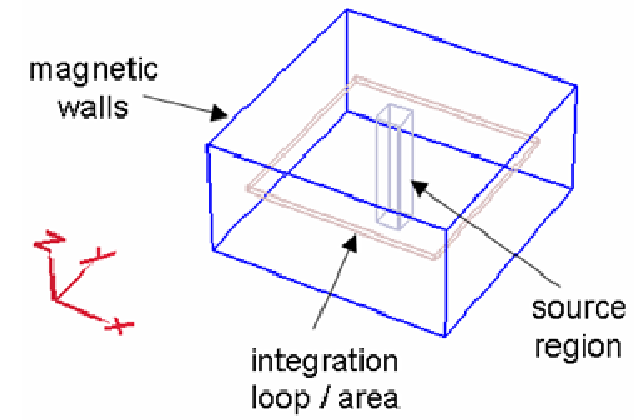

Gaussian

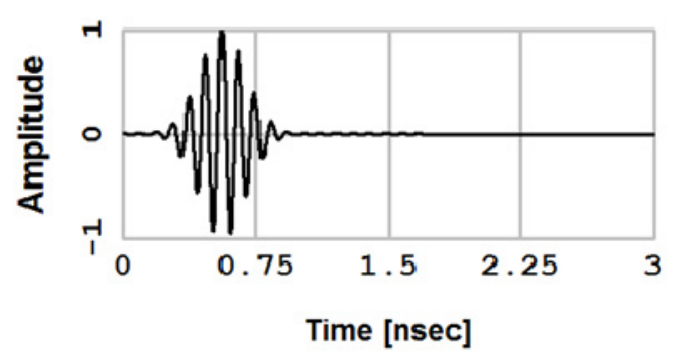

Figure 2: A magnetic cavity $\left(20 \times 20 \times 10 \mathrm{~mm}^{3}\right)$ used to simulate Faraday's Law. Also shown in the figure is a transient signal, a cosine modulated Gaussian pulse, $f_{\mathrm{o}}=10.75 \mathrm{GHz}$, bandwidth $=2 \mathrm{GHz}$, injected at the source region to induce an oscillating field. 

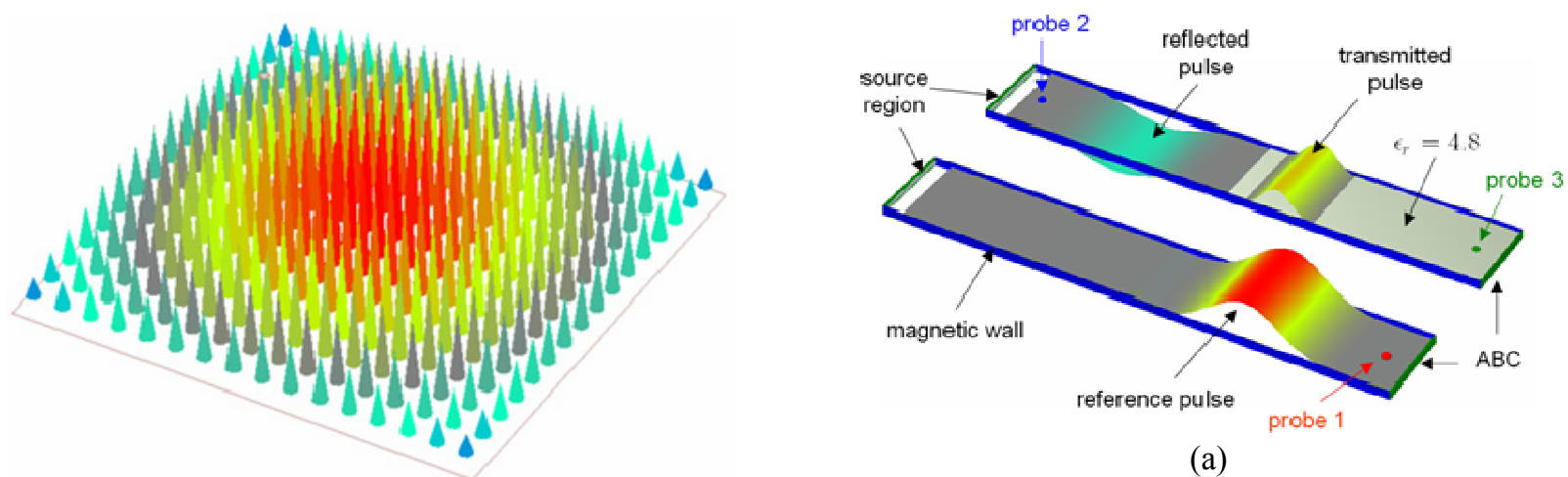

(a)

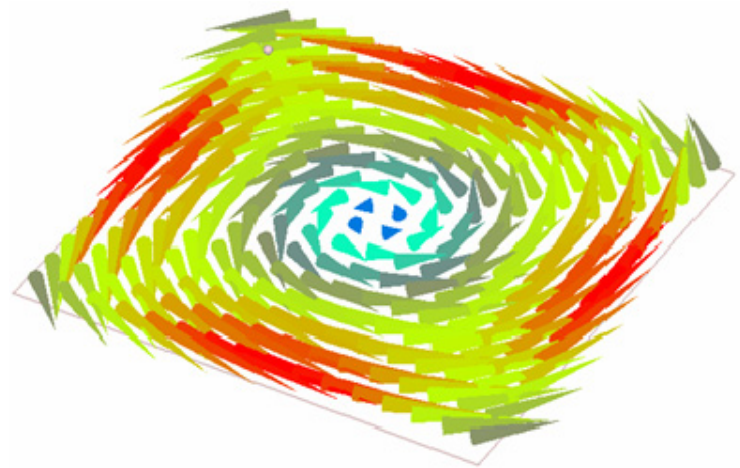

Figure 3: Top - magnetic field, $H_{z}$. Bottom - the associated transverse electric field, $E_{x y}$.

\subsection{Wave Phenomena}

Transverse electromagnetic (TEM) wave propagation, reflection and transmission can be simulated easily with $\mathcal{M E F i S \mathcal { T }}$. An efficient way to simulate these kinds of wave behaviour is to make use of the parallel plate waveguide. Figure 4 is a sequence of result that illustrates these wave behaviours obtained with two parallel plate waveguide. The first waveguide is half filled with air and half filled with dielectric, $\epsilon_{r}=4.8$; the second one is a reference structure. Figure $4 \mathrm{a}$ shows a snapshot of the wave after the incident pulse has been separated into incident and transmitted pulses. The static image in figure $4 \mathrm{a}$ cannot show the dynamic behaviour of the scattering process at the dielectric interface. However, the figure does reveal the transmitted pulse propagates slower than the reference pulse. Figure $4 \mathrm{~b}$ depicts the signal magnitudes recorded as a function of time when the pulses traveling down the structures. The magnitudes of the reflected and transmitted pulses are in agreement with the theoretical calculations, $\Gamma=\frac{\left(1-\sqrt{\epsilon_{r}}\right)}{\left(1+\sqrt{\epsilon_{r}}\right)}=-0.374$ and $T=1+\Gamma=0.626$.

Figure $4 \mathrm{c}$ shows the periodical nature of the field when the waveguide pair is excited with a $10 \mathrm{GHz}$

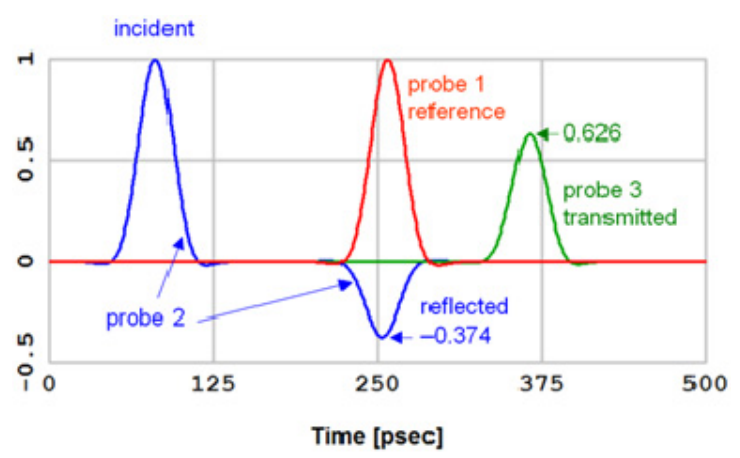

(b)

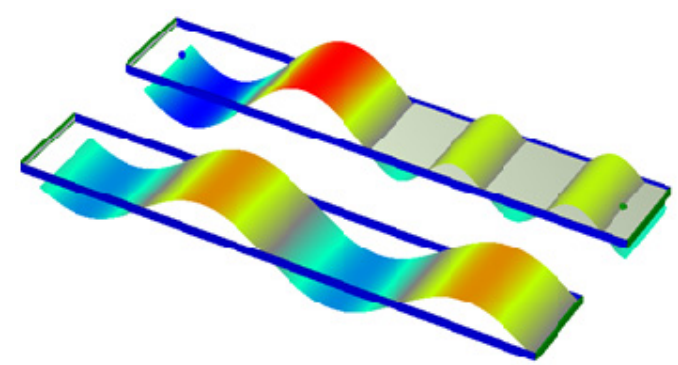

(c)

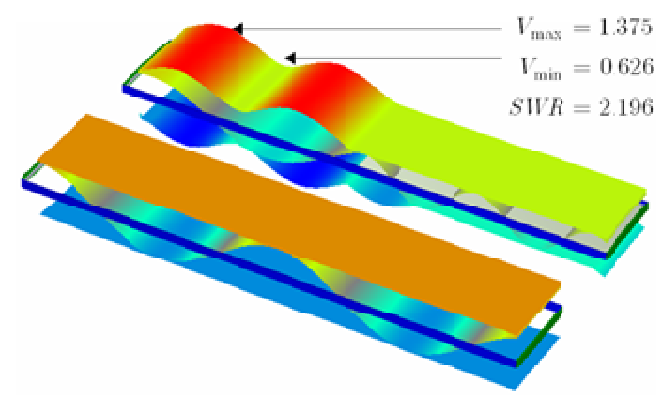

(d)

Figure 4: (a) A snapshot of the field distribution after the incident pulse has been scattered. (b) Signal magnitudes at the probe locations as a function of time. (c) A snapshot of the field when the waveguide pair is excited with a sine wave. (d) The time varyingwaves sandwiched inside their standing-wave envelopes. 
sine wave. It can be observed from the figure that the amplitude of the wave in the first waveguide is larger than that of the second one. This is due to the existence of a standing wave in the first structure. Figure $4 \mathrm{~d}$ shows the time varying-waves sandwiched inside their standing-wave envelopes.

\subsection{Phase And Group Velocities}

$\mathcal{M E F} \dot{\mathcal{S}} \mathcal{T}$ o has built-in metamaterial models [5 - 7] that possess negative permittivity and permeability at designed frequency. For TEM wave traveling in the metamaterial, the phase velocity is $v_{p}=1 / \sqrt{\epsilon_{r} \mu_{r}}$. If $\epsilon_{r}$ and $\mu_{r}$ are both negative, the phase velocity could be negative as well. Figure 5 depicts an airmetamaterial-air configuration. If the impedance of the metamaterial equal $\eta_{o}=377 \Omega$, then the wave impinging from the left should penetrate the airmetamaterial interface without reflection. Although the impedance at the interface is matched, the phase velocity in the metamaterial is negative; a dynamic field animation would show that the wave-fronts on both sides of interface (1) travel towards the interface while the wave-fronts on both sides of interface (2) move away from the interface, figure 5. Moreover, $\Gamma=0$ implies energy must continuously move forward, i.e. the group velocity in the metamaterial must be positive. This is illustrated in figure 6 via a multi-tone excitation.

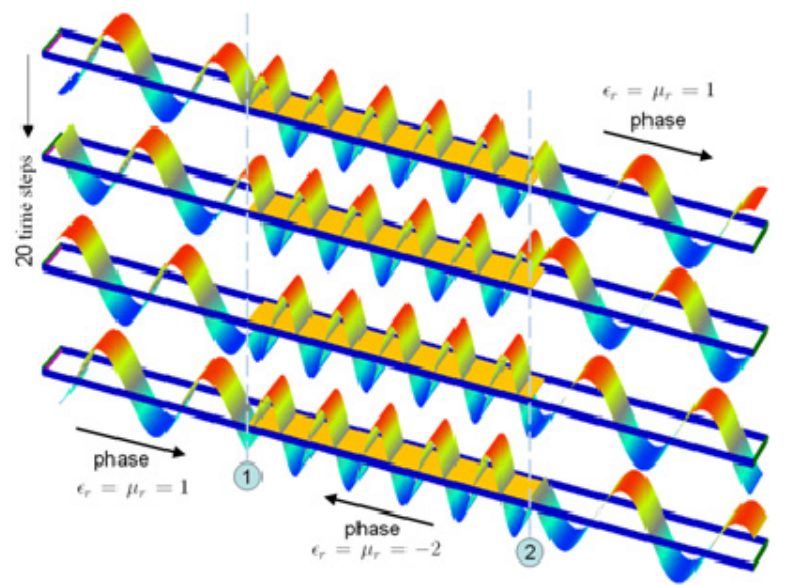

Figure 5: TEM wave propagating through a slab of metamaterial $\left(\epsilon_{\mathrm{r}}=\mu_{\mathrm{r}}=-2\right)$. The images depict a wave moving from left to right. A monochromatic wave moving from left to right; the wave-fronts on both sides of interface (1) travel towards the interface while the wave-fronts on both sides of interface (2) move away from the interface.

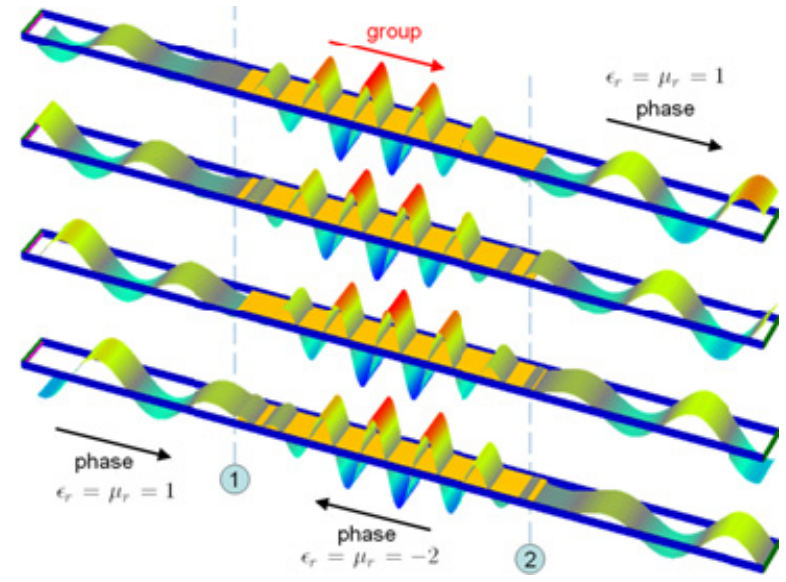

Figure 6: A multi-tone wave moving from left to right.

The successive locations of the wave envelope revealed the group velocity in the metamaterial is +ve.

\subsection{Focusing Effect}

If the phase velocity in the metamaterial is negative then the refractive index of metamaterial, which is the reciprocal of the phase velocity, must also be negative. Substituting a negative refractive index into the Snell's Law would obtain a negative angle of refraction [9, 10]. Hence a slab of metamaterial can focus electromagnetic wave much like a convex lens. Due to the focusing ability of the metamaterial, a $90^{\circ}$ prism made of metamaterial can produce a more focused reflected wave than a prism made of regular material. This effect is shown in figure 7.

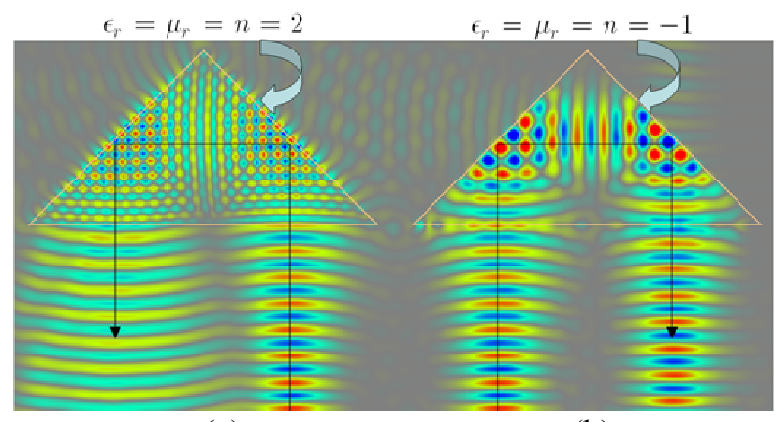

(a)

(b)

Figure 7: A Gaussian beam reflected by two different $90^{\circ}$ prisms. (a) refractive index $=2$, (b) refractive index $=-1$. The second prism can produce a more focused reflected beam. 


\section{Conclusion}

A sequence of numerical experiments that illustrates the dynamic behaviour of electromagnetic wave in various propagation situations has been presented. These experiments can be easily conducted on a home computer using $\mathcal{M E F} \mathcal{S}^{\mathrm{S}} \mathcal{T}$ - $3 \mathcal{D} \mathcal{N} O \mathcal{V} \mathcal{A}$. Students and educators interested in using the software may download it from Faustus Scientific Corporation (www.faustcorp.com) or from the author's website (www.cerl.ece.uvic.ca/poman/Courses/ELEC340/ELE C340.htm).

\section{Acknowledgement}

The author wishes to acknowledge the financial support from the National Science and Engineering Research Council of Canada as well as the invaluable contribution by Professor Wolfgang J.R. Hoefer in creating these examples. Last but not least, the generosity of Faustus Scientific Corporation for releasing the software as a free package to benefit the engineering electromagnetics community.

\section{References}

[1] P.B. Johns, A Symmetrical Condensed Node for the TLM Method, IEEE Trans. Microwave Theory and Tech. vol. 35, no. 4, pp. 370-377, April 1987.

[2] P.P.M. So, Eswarappa and W.J.R. Hoefer, A Two-Dimensional Transmission Line Matrix Microwave Field Simulator Using New Concepts and Procedures, IEEE Trans. MTT, vol.37, no.12, pp.1877-1898, December 1989.

[3] P.P.M. So, and W.J.R. Hoefer, 3D-TLM time domain electromagnetic wave simulator for microwave circuit modeling, IEEE MTT-S, vol.2, Boston, Massachusetts, pp.631-634, June 1991.

[4] W.J.R. Hoefer and P.P.M. So, A Time-Domain Virtual Electromagnetics Laboratory for Microwave Engineering Education, IEEE Trans. on Microwave Theory and Techniques, vol.51, no.4, pp. 1318-1325, April 2003.

[5] G.V. Eleftheriades, A.K. Iyer and P.C. Kremer, Planar negative refractive index media using periodically L-C loaded transmission lines, IEEE Trans. MTT, vol.50, no.12, pp.2702-2712, December 2002.

[6] Sanada, C. Caloz and T. Itoh, Planar distributed structures with negative refractive index, IEEE Trans. MTT, vol.52, no.4, pp.1252-1263, April 2004.

[7] P.P.M. So, H. Du and W.J.R. Hoefer, Modeling of metamaterials with negative refractive index using 2D shunt and 3D SCN TLM networks, IEEE Trans. MTT, vol.53, no.4, pp.1496-1505, April 2005.

[8] V. G. Veselago, The electrodynamics of substances with simultaneously negative values of $\varepsilon$ and $\mu$, Sov. Phys.-Usp., vol. 10, no. 4, pp.509-514, Jan.-Feb. 1968.

[9] J. B. Pendry, A. J. Holden, D. J. Robins, and W. J. Stewart, Magnetism from conductors and enhanced nonlinear phenomena, IEEE Trans. MTT, vol. 47, no. 11, pp. 2075-2084, Nov. 1999.

[10] D. R. Smith, W. J. Padilla, D. C. Vier, S. C. Nemat-Nasser, and S. Schultz, Composite medium with simultaneously negative permeability and permittivity, Phys. Rev. Lett., vol. 84, no. 18, pp. 4184-4187, May 2000. 